\title{
Gray Level Image Threshold Using Neutrosophic Certainty ${ }^{1}$
}

\author{
PATRASCU, Vasile \\ Research Center in Electrical Engineering, \\ Electronics and Information Technology \\ Valahia University of Targoviste \\ e-mail: patrascu.v@gmail.com
}

\begin{abstract}
This article presents a new method of segmenting images with gray levels. The method is based on determining several thresholds for separation of gray levels. The determination of these thresholds is done using the certainty of the neutrosophic information. The concept of this method can be stated simply: to choose the local maximums for the neutrosophic certainty.
\end{abstract}

Keywords: image segmentation, gray level thresholds, neutrosophic information, neutrosophic certainty

ACM/AMS Classification: 68U10, 62H35

\section{Introduction}

Segmentation of images with gray levels is a procedure of determining several subsets for the gray levels existing in that image and then, approximating the gray levels with the average of the intervals of which they are part. These subsets are intervals of real numbers, usually contained in the range $[0,225]$ or $[0,1]$. The separation of these intervals is done by determining optimal thresholds. In this article, the determination of these thresholds is constructed using elements from the neutrosophic theory of information representation.

Next, the article has the following structure: Section 2 presents an introduction for the neutrosophic representation of information; Section 3 presents the metric for gray levels, subsequently used to define a measure of the similarity of gray levels; Section 4 shows a presentation of the neutrosophic certainty used as an objective function to determine the separation thresholds; Section 5 presents the neutrosophication procedure of a gray level image; Section 6 presents the segmentation method; Section 7 presents some experimental results; Section 8 presents the conclusions. The article ends with the section of references.

\footnotetext{
${ }^{1}$ The Conference on Applied Mathematics and Informatics, CAMAI 2019, Bucharest, Romania, November 15-18, 2019
} 


\section{The Neutrosophic Information Representation}

The neutrosophic information is described by three parameters $T \in[0,1]$, $F \in[0,1], I \in[0,1]$ where $T$ is the degree of truth, $F$ is the degree of falsity and $I$ is the degree of indeterminacy or degree of neutrality [2], [3], [9], [10]. Neutrosophic representation of information is an extension of fuzzy representation [12] and intuitionistic fuzzy representation [1]. The neutrosophic information is incomplete when $T+I+F<1$, is complete when $T+I+F=1$ and it is inconsistent when $T+I+F>1$.

The order relation between two neutrosophic information is define by:

$$
\left(T_{p}, I_{p}, F_{p}\right) \succeq\left(T_{q}, I_{q}, F_{q}\right)
$$

if and only if

$$
\begin{gathered}
\Delta T \geq 0 \\
\Delta T \geq \Delta F \\
\Delta T \geq \Delta F+\Delta I
\end{gathered}
$$

where

$$
\begin{aligned}
\Delta T & =T_{p}-T_{q} \\
\Delta F & =F_{p}-T_{q} \\
\Delta I & =I_{p}-I_{q}
\end{aligned}
$$

There are the following inequalities for the vertices of the neutrosophic cube, namely the unit cube $[0,1] \times[0,1] \times[0,1]$ :

$$
\begin{aligned}
& (1,0,0)>((1,1,0)>(1,0,1)>(0,0,0)>(0,1,0)>(0,0,1)>(0,1,1) \\
& (1,0,0)>((1,1,0)>(1,0,1)>(1,1,1)>(0,1,0)>(0,0,1)>(0,1,1)
\end{aligned}
$$

The neutrosophic information $p=(0,0,0)$ and $q=(1,1,1)$ are not comparable because $\Delta T<0$ while $\Delta T=\Delta F$ and $\Delta T>\Delta F+\Delta I$.

In the next we will define the two main binary operations for neutrosophic information: the join and the meet. We will use the following binary operations for real numbers:

$$
\begin{aligned}
& x \vee y=\max (x, y) \\
& x \wedge y=\min (x, y)
\end{aligned}
$$

The join :

We will use for the join the symbol $\cup$. For two triplets $p=\left(T_{p}, I_{p}, F_{p}\right)$ and $q=\left(T_{q}, I_{q}, F_{q}\right)$ the join operation is define by the following three relations:

$$
\begin{gathered}
T_{p \cup q}=T_{p} \vee T_{q} \\
F_{p \cup q}=T_{p} \vee T_{q}-\left(T_{p}-F_{p}\right) \vee\left(T_{q}-F_{q}\right) \\
I_{p \cup q}=\left(T_{p}-F_{p}\right) \vee\left(T_{q}-F_{q}\right)-\left(T_{p}-F_{p}-I_{p}\right) \vee\left(T_{q}-F_{q}-I_{q}\right)
\end{gathered}
$$


The meet :

We will use for the meet the symbol $\cap$. For two triplets $p=\left(T_{p}, I_{p}, F_{p}\right)$ and $q=\left(T_{q}, I_{q}, F_{q}\right)$ the meet operation is define by the following three relations:

$$
\begin{gathered}
T_{p \cap q}=T_{p} \wedge T_{q} \\
F_{p \cap q}=T_{p} \wedge T_{q}-\left(T_{p}-F_{p}\right) \wedge\left(T_{q}-F_{q}\right) \\
I_{p \cap q}=\left(T_{p}-F_{p}\right) \wedge\left(T_{q}-F_{q}\right)-\left(T_{p}-F_{p}-I_{p}\right) \wedge\left(T_{q}-F_{q}-I_{q}\right)
\end{gathered}
$$

At the end of this section, we will define three unary operations for neutrosophic information. These operations will be used for neutrosophic certainty properties description.

The complement is define by :

$$
(T, I, F)_{c}=(F, I, T)
$$

The negation is define by :

$$
(T, I, F)_{n}=(1-T, I, 1-F)
$$

The dual is define by:

$$
(T, I, F)_{d}=(1-F, I, 1-T)
$$

\section{The Gray Level Similarity Function}

In this section, we will show, how was constructed the gray level similarity function that was used for the proposed segmentation method. The interval $[0,1]$ was considered for the range of the gray levels. In order to compute the gray level similarity, we will use the negation of dissimilarity and for the dissimilarity between two gray levels $x, y \in[0,1]$, we will use the following metric [6], [7]:

$$
\begin{aligned}
& d:[0,1] \times[0,1] \rightarrow[0,1] \\
& \qquad d(x, y)=\frac{2|x-y|}{1+|x-0.5|+|y-0.5|}
\end{aligned}
$$

We will show how can we obtain the metric (21). We consider the following distance $D: R^{2} \rightarrow[0,1]$, defined by [11]:

$$
D(x, y)=\frac{|x-y|}{1+|x|+|y|}
$$

$D(x, y)$ is a metric because it satisfies the following conditions:

(i) $D(x, y)=D(y, x)$ 
(ii) $D(x, y)=0$ if $x=y$

(iii) $D(x, y)+D(y, z) \geq D(x, z)$

It is obvious that the distance $D$ verifies the conditions (i) and (ii). In the next, we will prove that it verifies the triangle inequality:

$$
\frac{|x-y|}{1+|x|+|y|}+\frac{|y-z|}{1+|y|+|z|} \geq \frac{|x-z|}{1+|x|+|z|}
$$

This is obtained using the following three inequalities [4]:

$$
\begin{gathered}
|x-y|+|y-z| \geq|x-z| \\
|x-y| \cdot|z|+|y-z| \cdot|x| \geq|x-z| \cdot|z| \\
\frac{|x-y|}{|x|+|y|}+\frac{|y-z|}{|y|+|z|} \geq \frac{|x-z|}{|x|+|z|}
\end{gathered}
$$

Using the metric $D$ we define the metric $d$ by:

$$
d(x, y)=2 D(x-0.5, y-0.5)
$$

It results:

$$
d(x, y)=\frac{2|x-y|}{1+|x-0.5|+|y-0.5|}
$$

One obtains the similarity $s(x, y)$ between two gray levels $x$ and $y$ with the negation of $d(x, y)$, namely:

$$
s(x, y)=1-\frac{2|x-y|}{1+|x-0.5|+|y-0.5|}
$$

With this similarity, we will construct the neutrosophic information associated with each gray level $x$ and related to the threshold $t$.

\section{The Certainty for Neutrosophic Information}

The neutrosophic certainty is a feature of the neutrosophic information that shows the similarity between the triplet $(T, I, F)$ and the set $V=$ $\{(1,0,0),(0,0,1)\}$. Thereby, the certainty of the neutrosophic information increases when the point $(T, I, F)$ approaches one of the points of the set $V$. The certainty is maximum when $(T, I, F) \in V$. Because the neutrosophic uncertainty is maximum when $T=F$, the neutrosophic certainty must be minimal in this case. The certainty must be the same for the information $(T, I, F)$, for the complement of information $(F, I, T)$, for the negation of information $(1-T, I, 1-F)$ and in the same time for its dual, namely $(1-F, I, 1-T)$. 
As a result, in the space $(T, I, F)$ the neutrosophic certainty must verify the following conditions [5]:

i) maximality

$$
g(1,0,0)=g(0,0,1)=1
$$

ii) minimality

$$
g(T, I, T)=g(F, I, F)=0
$$

iii) symmetry

$$
g(T, I, F)=g(F, I, T)=g(1-T, I, 1-F)=g(1-F, I, 1-T)
$$

iv) monotonicity

$$
g\left(T_{1}, I_{1}, F_{1}\right) \leq g\left(T_{2}, I_{2}, F_{2}\right)
$$

if $\left|T_{1}-F_{1}\right| \leq\left|T_{2}-F_{2}\right|,\left|T_{1}+F_{1}-1\right| \geq\left|T_{2}+F_{2}-1\right|, I_{1} \geq I_{2}$.

The property (iv) shows that the certainty increases with $|T-F|$, decreases with $|T+F-1|$ and decreases with $I$.

From (iv) it results that $g(T, I, F) \in[0,1]$ because $g(T, I, F) \leq g(1,0,0)$ and $g(T, I, F) \geq g(0,1,0)$.

In this paper, the formula presented in [5] is used as a measure for the neutrosophic certainty.

$$
g(T, I, F)=\frac{|T-F|}{1+I+|T+F-1|}
$$

\section{The Neutrosophic Information Construction}

Any gray level image is defined by the triplet $(\Omega, A, h)$ where $\Omega=$ $\{1,2, \ldots, m\} \times\{1,2, \ldots, n\}$ represents the image domain, $A=[0,1]$ represents the gray level range, $h: \Omega \rightarrow A$ is the function that define the image. We consider the multiset $X=h(\Omega)$ and we denote

$$
\begin{gathered}
x_{m}=\min (X) \\
\left.x_{M}=\max (X)\right)
\end{gathered}
$$

and take a point $t \in\left(x_{m}, x_{M}\right)$. Also, we define the following two multisets $X_{1}$ and $X_{2}$ by:

$$
\begin{aligned}
& X_{1}=\{x \in X \mid x \leq t\} \\
& X_{2}=\{x \in X \mid x \geq t\}
\end{aligned}
$$

Then, we calculate the averages $v_{1}$ and $v_{2}$ with formulas: 


$$
\begin{aligned}
& v_{1}(t)=\frac{1}{\operatorname{card}\left(X_{1}\right)} \sum_{x \in X_{1}} x \\
& v_{2}(t)=\frac{1}{\operatorname{card}\left(X_{2}\right)} \sum_{x \in X_{2}} x
\end{aligned}
$$

For calculation the similarity between two gray levels $x, y \in[0,1]$ we will use the similarity function $s$ presented before, namely:

$$
\begin{aligned}
s:[0,1] \times[0,1] & \rightarrow[0,1], \\
& s(x, y)=1-\frac{2|x-y|}{1+|x-0.5|+|y-0.5|}
\end{aligned}
$$

We will calculate the similarities between each gray level $x$ and the averages $v_{1}, v_{2}$ and the threshold $t$. It results $s\left(x, v_{1}\right), s\left(x, v_{2}\right)$ and $s(x, t)$.

$$
\begin{gathered}
s\left(x, v_{1}\right)=1-\frac{2\left|x-v_{1}(t)\right|}{1+|x-0.5|+\left|v_{1}(t)-0.5\right|} \\
s\left(x, v_{2}\right)=1-\frac{2\left|x-v_{2}(t)\right|}{1+|x-0.5|+\left|v_{2}(t)-0.5\right|} \\
s(x, t)=1-\frac{2|x-t|}{1+|x-0.5|+|t-0.5|}
\end{gathered}
$$

Also, we define the similarity between the gray level $x$ and the pair $\left(v_{1}, v_{2}\right)$ with the following formula:

$$
s_{v}\left(x, v_{1}, v_{2}\right)=\max \left(s\left(x, v_{1}\right), s\left(x, v_{2}\right)\right)
$$

With these four similarities, we will construct the neutrosophic information associated with each gray level $x$ and related to the threshold $t$ : the degree of truth $T(x, t)$, the degree of falsity $F(x, t)$ and the degree of neutrality $I(x, t)[8]$.

The degree of truth:

$$
T(x, t)=\frac{s\left(x, v_{1}\right) \cdot\left(1-s\left(x, v_{2}\right)\right)}{1-s\left(x, v_{1}\right) \cdot s\left(x, v_{2}\right)}
$$

The degree of falsity:

$$
F(x, t)=\frac{s\left(x, v_{2}\right) \cdot\left(1-s\left(x, v_{1}\right)\right)}{1-s\left(x, v_{1}\right) \cdot s\left(x, v_{2}\right)}
$$

The degree of neutrality:

$$
I(x, t)=\frac{s(x, t) \cdot\left(1-s_{v}\left(x, v_{1}, v_{2}\right)\right)}{1-s(x, t) \cdot s_{v}\left(x, v_{1}, v_{2}\right)}
$$


It results the following formula for the neutrosophic certainty associated to each gray level $x$ and related to the threshold $t$.

$$
g(x, t)=\frac{|T(x, t)-F(x, t)|}{1+I(x, t)+|T(x, t)+F(x, t)-1|}
$$

\section{The Segmentation Method}

Firstly, we will construct the set of the possible values for the thresholds. This is the following set:

$$
V_{t h}=\left\{\frac{1}{Q}, \frac{2}{Q}, \ldots, \frac{Q-1}{Q}\right\}
$$

where $Q$ is a natural number and $Q \geq 100$. In addition, we will select those values that are in the range $\left(x_{m}, x_{M}\right)$ defined by (35) and (36). Thus, for each $t \in\left(x_{m}, x_{M}\right) \cap\left\{\frac{1}{Q}, \frac{2}{Q}, \ldots, \frac{Q-1}{Q}\right\}$, we calculate the certainty average for the fuzzy sets defined by the neutrosophic components $T$ and $F$.

It results the partial certainty functions: $g_{T}(t), g_{F}(t)$.

$$
\begin{aligned}
& g_{T}(t)=\frac{\sum_{x \in X} T(x, t) \cdot g(x, t)}{\sum_{x \in X} T(x)} \\
& g_{F}(t)=\frac{\sum_{x \in X} F(x, t) \cdot g(x, t)}{\sum_{x \in X} F(x, t)}
\end{aligned}
$$

Then, we define the total certainty $G(t)$, which is calculated with the sum of the partial certainties calculated above.

$$
\left.G(t)=g_{T}(t)\right)+g_{F}(t)
$$

The segmentation thresholds are the local maximum points of the total certainty.

We suppose that at the end, we obtained $k$ thresholds $t_{1}, t_{2}, \ldots, t_{k}$. In this way, we obtained $k+1$ subsets $w_{1}=\left(x_{m}, t_{1}\right) \cap X, \ldots, w_{i}=\left(t_{i-1}, t_{i}\right) \cap X, \ldots$, $w_{k+1}=\left(t_{k}, x_{M}\right) \cap X$. For each subset $w_{i}$, we will calculate its average $c_{i}$ with the formulas:

$$
c_{i}=\frac{1}{\operatorname{card}\left(w_{i}\right)} \sum_{x \in w_{i}} x
$$

Finally, the gray levels in each subset $w_{i}$ are replaced by the average $c_{i}$ of that subset. 


\section{Experimental Results}

To test the properties of this new method, we implement it in four gray level images, as shown respectively in figures 1(a), 2(a), 3(a) and 4(a). The proposed method was applied for segmentation of the following images: Fz0, Geometric, Lena and Bobby. The obtained results and the graphic of the certainty functions can be seen in figures 1(b,c), 2(b,c), 3(b,c) and 4(b,c). The total certainty for image Fz0 has only one maximum for $t=0.49$. The averages obtained are $c_{1}=0.23$ and $c_{2}=0.76$. The total certainty for image Geometric has three local maximums for $t_{1}=0.33, t_{2}=0.52$ and $t_{3}=0.62$. The averages obtained are $c_{1}=0.19, c_{2}=0.42, c_{3}=0.60$ and $c_{4}=0.70$. The total certainty for image Lena has three local maximums for $t_{1}=0.31$, $t_{2}=0.73$ and $t_{3}=0.89$. The averages obtained are $c_{1}=0.22, c_{2}=0.53$, $c_{3}=0.80$ and $c_{4}=0.90$. The total certainty for image Bobby has two local maximums for $t_{1}=0.27$ and $t_{2}=0.74$. The averages obtained are $c_{1}=0.15$, $c_{2}=0.50$ and $c_{3}=0.82$.

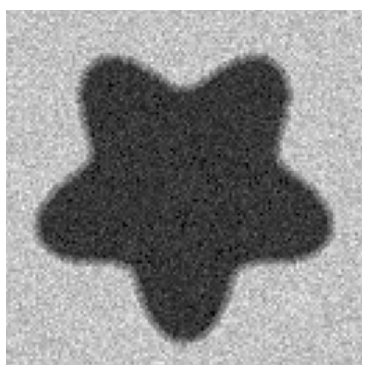

a)

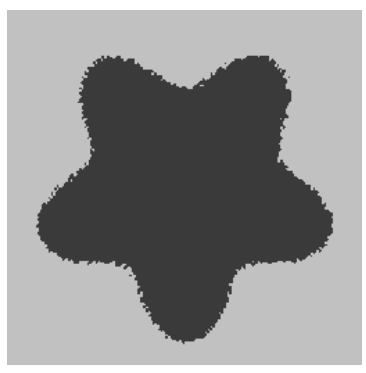

b )

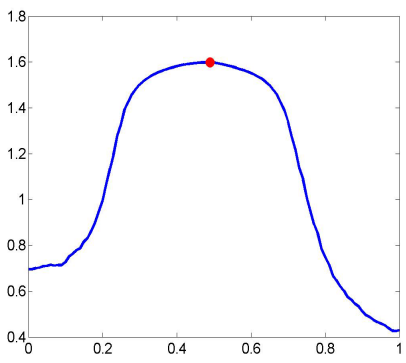

c)

Figure 1: The image Fz0 $(a)$. The segmented image with two gray levels $(b)$. The graphic of neutrosophic certainty and its local maximum $(c)$.

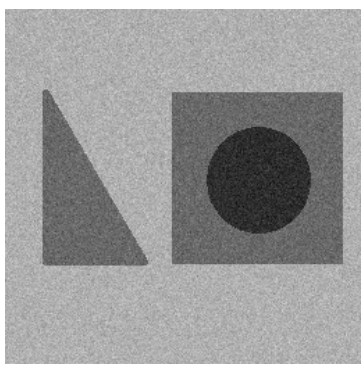

a)

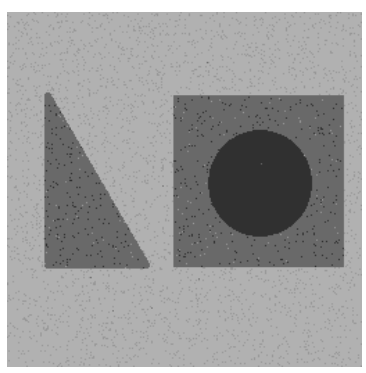

b)

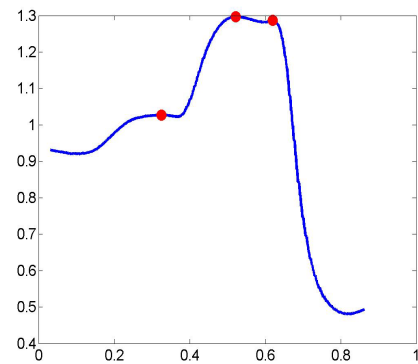

c)

Figure 2: The image Geometric $(a)$. The segmented image with four gray levels $(b)$. The graphic of neutrosophic certainty and its local maximums $(c)$. 


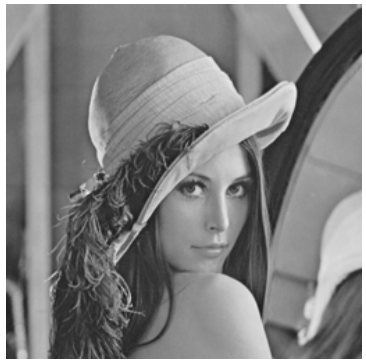

a)

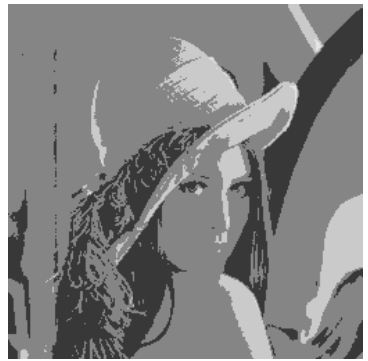

b)

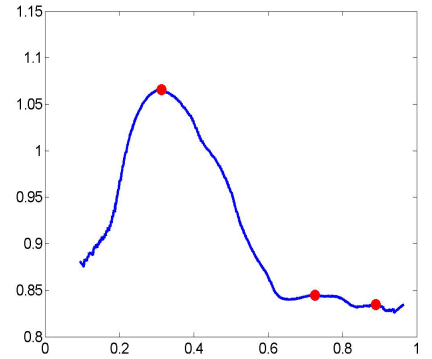

c)

Figure 3: The image Lena $(a)$. The segmented image with four gray levels (b). The graphic of neutrosophic certainty and its local maximums $(c)$.

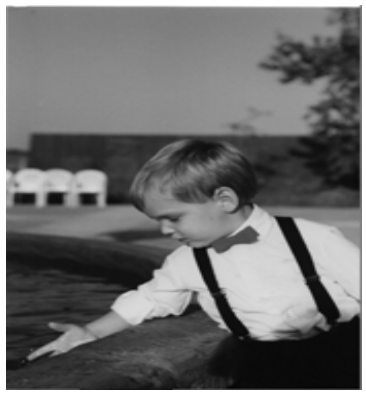

a)

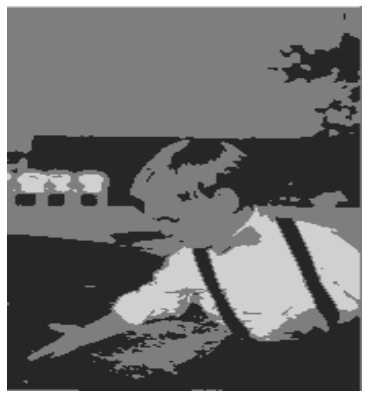

b)

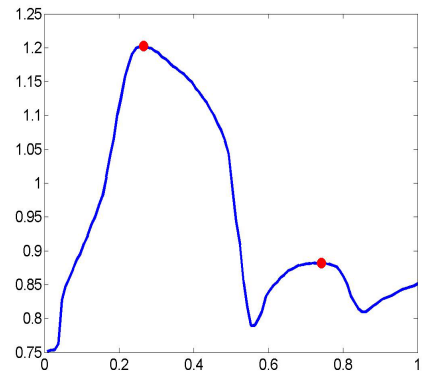

c)

Figure 4: The image Bobby $(a)$. The segmented image with three gray levels (b). The graphic of neutrosophic certainty and its local maximums $(c)$.

\section{Conclusions}

The article presents a method of segmenting images with gray levels, using the separation thresholds for intervals of gray levels. The separation thresholds are determined by the local maximum points of the neutrosophic certainty. In order to be able to use the neutrosophic certainty, it is presented the way of attaching a neutrosophic information to a gray level. Obviously, this attached neutrosophic information is related to the considered threshold.

The presented method may provide multiple separation thresholds. The experimental results show us that, indeed, the local maximum points of the neutrosophic certainty can provide separation thresholds for the gray levels contained in an image. Also, the experimental results show us that it is possible to segment the color images, using the presented method.

In addition, this article falls into the category of articles that presents the possibility of using neutrosophical theory in engineering and in particular case in the image processing area. 


\section{References}

1. K. Atanassov. Intuitionistic Fuzzy sets. "Fuzzy Sets and Systems", 20, pp. 87-96, 1986.

2. G. Albeanu, Neutrosophic Computational Models - I, "Annals of Spiru Haret University", Mathematics-Informatics Series, Volume IX /, Issue 2, pp. 13-22, 2013.

3. G. Albeanu, Neutrosophic Computational Models - II, "Annals of Spiru Haret University", Mathematics-Informatics Series, Volume X, Issue 2, pp. 51-56, 2014.

4. M.S. Klamkin, A. Meir, Ptolemey's Inequality, Chordal Metric, Multiplicative Metric, "Pacific Journal of Mathematics", Vol. 101, No. 2, pp. 389-393, 1982.

5. V. Patrascu, Shannon Entropy for Neutrosophic Information, "doi:10.13140/RG.2.2.32352.74244", arXiv:1810.00748, September, 2018.

6. V. Patrascu, A Novel Penta-Valued Descriptor for Color Clustering, "The 6th International Conference on Image and Signal Processing", ICISP 2014, Cherbourg, Normandy, France, June 30 - July 2, 2014, Volume: Image and Signal Processing, Lecture Notes in Computer Science, Volume 8509, Springer International Publishing Switzerland, pp. 173182, 2014.

7. V. Patrascu, New Framework of HSL System Based Color Clustering Algorithm, "The 24th Midwest Artificial Intelligence and Cognitive Sciences Conference", MAICS 2013, April 13-14, 2013, New Albany, Indiana. USA, ceur-ws.org, Vol. 1348, pp. 85-91, 2013.

8. V. Patrascu, Gray Level Image Threshold Using Neutrosophic Shannon Entropy "The 3rd Conference on Recent Advances in Artificial Intelligence", RAAI 2019, Bucharest, Romania, June 28-30, 2019.

9. F. Smarandache, Neutrosophy, "arXiv:math/0010099", 11 Oct. 2000

10. F. Smarandache, An Introduction to the Neutrosophic Probability Applied in Quantum Physics, "arXiv:math/0010088", 10 oct 2000.

11. P. N. Yianilos, Normalized Forms for Two Common Metrics, Technical Memorandum, "NEC Research Institute", 91-082-3-902, 5 December 1991.

12. A. L. Zadeh. Fuzzy sets, "Information and Control", 8, pp. 338-353, 1965. 